\title{
A case of CHARGE syndrome featuring immunodeficiency and hypocalcemia
}

Yu Yun Son', Byeonghyeon Lee ${ }^{2}$, Chae-Ri Suh', Hyo-Kyoung Nam¹, Jung Hwa Lee'*, Young Sook Hong', and Joo Won Lee 'Department of Pediatrics, Korea University College of Medicine, Seoul, Korea

2Department of Biology, School of Life Sciences, KNU Creative BioResearch Group (BK21 plus project), Kyungpook National University, Daegu, Korea

CHARGE syndrome (coloboma, heart defects, atresia choanae, retarded growth and development, genital hypoplasia, and ear abnormalities) is characterized by multiple malformations and is diagnosed using distinct consensus criteria. Mutations in the gene encoding chromodomain helicase DNA-binding protein 7 (CHD7) are the major cause of CHARGE syndrome. Clinical features of CHARGE syndrome considerably overlap those of 22q11.2 deletion syndrome. Of these features, immunodeficiency and hypocalcemia are frequently reported in patients with 22q11.2 deletion syndrome but are rarely reported in patients with CHARGE syndrome. In this report, we have described the case of a patient with typical phenotypes of 22q11.2 deletion syndrome but without the proven chromosome microdeletion. Mutation analysis of $\mathrm{CHD}$ identified a pathogenic mutation (c.2238+1G>A) in this patient. To our knowledge, this is the first case of CHARGE syndrome with immunodeficiency and hypocalcemia in Korea. Our observations suggest that mutation analysis of $C H D 7$ should be performed for patients showing the typical phenotypes of 22q11.2 deletion syndrome but lacking the proven chromosome microdeletion.

Key words: CHD protein, Chromosome 22q11.2 deletion syndrome, Immune deficiency.

\section{Introduction}

CHARGE syndrome is characterized by multiple congenital anomalies and affects approximately 1 child per 10,000 live births $[1,2]$. It is named after its 6 cardinal clinical features: coloboma, heart malformation, atresia choanae, retarded growth and development, genital hypoplasia, and ear abnormalities. Mutations in the gene encoding chromodomain helicase DNA-binding protein 7 (CHD7) are responsible for the development of CHARGE syndrome. Disease-causing mutations in CHD7 are found in 60-70\% patients with CHARGE syndrome [3-5]. Although CHARGE syndrome can be clinically diagnosed using criteria put forth by Verloes [1], patients with CHARGE syndrome show varying phenotypes that considerably overlap those of other syndromes such as Kallmann syndrome, VACTERL association (vertebral anomalies, anal atresia, cardiac defects, tracheoesophageal fistula, esophageal atresia, renal anomalies, and limb defects), and 22q11.2 deletion syndrome $[3,6,7]$.

Chromosome 22q11.2 deletion is observed in majority of patients with DiGeorge syndrome [8]. Patients with DiGeorge syndrome show typical features such as conotruncal cardiac anomaly, abnormal face, thymic hypoplasia, cleft palate, and hypocalcemia, which have been given an acronym CATCH22 [9]. DiGeorge syndrome is the cause of congenital

\footnotetext{
Received: 6 May 2015, Revised: 27 May 2015, Accepted: 11 June 2015, Published: 30 June 2015

*Corresponding author: Jung Hwa Lee, M.D.

Department of Pediatrics, Korea University College of Medicine, 73 Inchon-ro, Seongbuk-gu, Seoul 136-705, Korea.

Tel: +82-2-2626-1229, Fax: +82-2-2626-1249, E-mail: leejmd@chollian.net

Conflict of interest: We declare that we do not have any conflicts of interests.

(c) This is an open-access article distributed under the terms of the Creative Commons Attribution Non-Commercial License (http://creativecommons.org/licenses/by-nc/4.0/) which permits unrestricted non-commercial use, distribution, and reproduction in any medium, provided the original work is properly cited.

(c) Copyright 2015 by the Korean Society of Medical Genetics

www.e-kjgm.org
} 
immunodeficiency in Korea, with a prevalence rate of 0.5 per million [10]. Auditory abnormality is the main manifestation of CHARGE syndrome. Immunodeficiency and hypocalcemia are rarely reported and have not been previously described in Korean patients with CHARGE syndrome [3].

Here, we report the case of a patient with CHARGE syndrome who also developed immunodeficiency and hypocalcemia.

\section{Case}

A 58-day-old girl was transferred to our hospital because of recurrent tetany. She was born to a healthy mother through an emergency cesarean section because of fetal deceleration at 39 weeks of gestation. Her Apgar scores were 6 and 7 at 1 and 5 minutes, respectively. Her birth weight, height, and head circumference were 2,960 g (25th-50th percentile), 50 $\mathrm{cm}$ (50th-75th percentile), and $34 \mathrm{~cm}$ (50th-75th percentile), respectively. After birth, the girl was transferred to a tertiary hospital for further management. Biochemical analysis of her serum sample indicated hypocalcemia (ionized calcium, 3.01 $\mathrm{mg} / \mathrm{dL}$; total calcium, $7.3 \mathrm{mg} / \mathrm{dL}$ ), which was normalized by intravenous infusion of calcium. After this, her calcium level remained within the normal range without further treatment. Results of other neonatal screening tests, including auditory screening by measuring automated auditory brainstem response, were normal. Echocardiography showed an atrial septal defect of permanent foramen ovale type and small patent ductus arteriosus. The patient had developmental dysplasia of the hip that was treated using a Pavlik harness. She also had a high-arched palate with a soft tissue cleft. Because she showed a poor ability to suck, she was fed by gavage. She was discharged at 25 days of age.

Biochemical analysis of her serum sample performed at 58 days of age (when she was transferred to our hospital because of recurred tetany) indicated hypocalcemia (ionized calcium, $1.84 \mathrm{mg} / \mathrm{dL}$; total calcium, $3.9 \mathrm{mg} / \mathrm{dL}$ ), hyperphosphatemia (13.6 mg/dL), normal alkaline phosphatase level (259 IU/L), low 25-hydroxyvitamin D level (19.4 ng/mL), and low parathyroid hormone level (3.0 pg/mL). Her face was square, with a broad, flat nose, and she had micrognathia and a short neck. Her face became asymmetric while crying, suggesting right facial nerve palsy. Both her ears were low set and dysmorphic, with the left ear being mildly constricted and the right ear being cup shaped. Her parents were healthy and unrelated, and her only brother had no problems that required medical attention.

Auditory-evoked potential testing showed sensorineural deafness, and ophthalmic examination showed coloboma of the left eye. Abdominal ultrasonography showed hydronephrosis of both the kidneys. Chest radiography did not detect a thymic shadow. Her hypocalcemia was treated by intravenous infusion of calcium followed by oral administration of calcium and vitamin D. Because she was suspected of having $22 q 11.2$ deletion syndrome, we performed chromosomal analysis, array comparative genomic hybridization, and fluorescent in situ hybridization. The results of these analyses showed that she had a normal 46, XX karyotype, with benign copy number variations in chromosomes Xq27.2, Xq28, 12p13.31, and 22q11.22 and no deletion of chromosome 22q11.2. She still showed poor swallowing and was fed by gavage. She was discharged at 70 days of age after stabilization of her calcium levels.

At 5 months of age, she was re-admitted to our hospital because of pneumonia. Before admission, she experienced frequent aspiration even of saliva during tube feeding. On the seventh day of hospitalization, her pneumonia exacerbated to acute respiratory distress syndrome (ARDS) because of which she was given mechanical ventilation. Previously noted lymphadenitis on her left axillar after BCG vaccination became suppurative, and scattered papules on her left leg were newly developed. Tissue biopsy of the scattered papules showed chronic granulomatous inflammation. Because of the risk of BCG dissemination, she was treated using isoniazid, rifampin, and ethambutol. Her immune status was evaluated because she experienced repeated, unusual, and severe infection. Lymphocyte surface marker analysis showed normal to increased number of total lymphocytes (24-27\%), white blood cells $(3,500-5,100 / \mu \mathrm{L}), \mathrm{T}$ lymphocytes (900-2,800/ $\mu \mathrm{L}), \mathrm{B}$ lymphocytes $(1,500-2,500 / \mu \mathrm{L})$, and natural killer cells (500-1,000/ $\mu \mathrm{L})$; low number of CD4 cells $(260-550 / \mu \mathrm{L})$; and inverse ratio of CD4/CD8 cells (0.2-0.4). Immunoglobulin analysis showed low levels of normal immunoglobulin (Ig) G (231 mg/dL) and normal levels of $\operatorname{lgM}(33 \mathrm{mg} / \mathrm{dL})$ and $\lg \mathrm{A}(7$ $\mathrm{mg} / \mathrm{dL}$ ). After intensive care with antibiotics and intravenous immunoglobulins, her condition finally improved, and she was discharged at 8 months of age. An esophagogram obtained at the time of discharge did not show tracheoesophageal fistula or gastroesophageal reflux.

At 9 months of age, the girl was re-admitted to our hospital because of pneumonia. At that time, her body weight was 5.2 $\mathrm{kg}(<3 \mathrm{rd}$ percentile) and her height was $59 \mathrm{~cm}$ ( $<3$ rd percentile). She showed developmental delay such as inability to rotate her trunk and sit alone. Immunoglobulin analysis showed low $\lg \mathrm{G}$ level $(216 \mathrm{mg} / \mathrm{dL})$. She was given mechanical ventilation 
because of pneumonia with ARDS. Examination of tracheal aspirate detected continuous infection of parainfluenza virus and intermittent infection of Pseudomonas aeruginosa, Acinetobacter baumannii, methicillin-resistant Staphylococcus aureus, and Candida albicans. Treatment with appropriate antibiotics and antifungals as well as intravenous infusion of immunoglobulins could not treat the pneumonia. Finally, she died because of ventilator-associated pneumonia and sepsis at 13 months of age. Subsequent mutation analysis of $\mathrm{CHD7}$ showed a pathogenic c.2238+1G>A mutation (Fig. 1), which has been previously reported in patients with CHARGE syndrome.

\section{Discussion}

According to the Verloes criteria [1], our patient had at least atypical CHARGE syndrome satisfying one major (coloboma) and three minor (facial palsy, abnormal external ear, and heart defect) criteria. In addition, her clinical features were similar

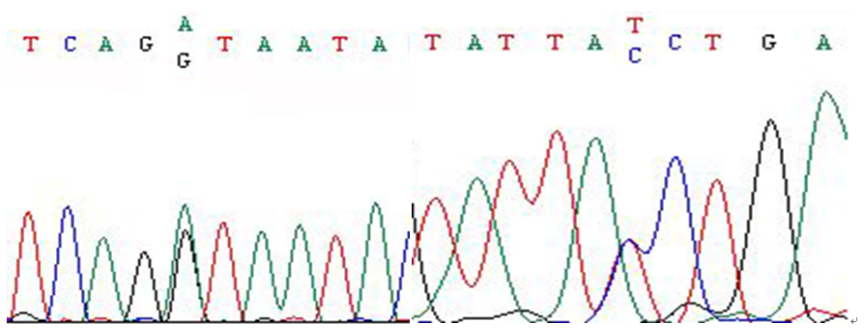

\section{Forward Reverse}

Fig. 1. Direct sequence analysis of $C H D 7$. Results of sequence analysis indicate the presence of $c .2238+1 \mathrm{G}>\mathrm{A}$ mutation in our patient. to those of patients with CATCH22 syndrome; however, she did not show chromosome 22q11.2 deletion. Because clinical features of CHARGE syndrome are similar to those of $22 q 11.2$ deletion syndrome, it is difficult to differentiate between the two syndromes on the basis of phenotypes alone (phenotypes of our patient are reported in Table 1). CHARGE syndrome can be easily diagnosed in boys because of the presence of genital hypoplasia. However, this feature was not observed in our female patient. Overlapping features of CHARGE and 22q11.2 deletion syndromes suggest a common neural crest defect and a mechanism that is partly explained by $T B X 1$, a major candidate gene involved in 22q11.2 deletion syndrome. This gene may be a functional target of CHD7. Alternatively, these 2 syndromes may involve common target genes in affected organs $[6,11,12]$. Most patients develop CHARGE syndrome sporadically because of de novo mutations in CHD7. Our patient seemed to be the only affected member in her family. Molecular examination of our patient showed a pathogenic splicing mutation in CHD7 and benign copy number variations in chromosomes 12p, 22q, and Xq but no 22q11.2 deletion $[4,13]$.

Although the frequency and nature of immunodeficiencies in patients with CHARGE syndrome have not been analyzed appropriately, these immunodeficiencies may be more common in patients with CHARGE syndrome than in patients with $22 q 11.2$ deletion syndrome $[6,7]$. The close association between lymphopenia and hypocalcemia in patients with CHARGE syndrome suggests that they develop because of impaired embryogenesis, which is similar to that observed in patients with 22q11.2 deletion syndrome [7]. Because most Korean infants

Table 1. Assessment of clinical features of CHARGE and 22q11.2 deletion syndromes in our patient

\begin{tabular}{lccc}
\hline \multicolumn{1}{c}{ Feature } & Our patient & CHARGE syndrome (frequency, \%) [6] & 22q11.2 deletion syndrome (frequency, \%) [6] \\
\hline Coloboma & + & $189 / 234(81)^{\mathrm{a}}$ & $4 / 612(1)$ \\
\hline Heart defect & + & $191 / 252(76)^{\mathrm{a}}$ & $667 / 868(77)^{\mathrm{a}}$ \\
\hline Atresia choanae & - & $99 / 179(55)$ & $6 / 611(1)^{\mathrm{a}}$ \\
Genital hypoplasia & $?$ & $118 / 145(81)$ & $88 / 698(13)$ \\
External ear anomaly & + & $224 / 231(97)^{\mathrm{a}}$ & $565 / 747(76)^{\mathrm{a}}$ \\
\hline Cleft lip and/or palate & + & $79 / 163(48)$ & $215 / 719(30)$ \\
\hline Thymic hypoplasia & + & - & $169 / 544(31)^{\mathrm{a}}$ \\
Hypoparathyroidism & + & - & $343 / 536(64)^{\mathrm{a}}$ \\
\hline Hearing loss & + & $183 / 192(95)^{\mathrm{a}}$ & $240 / 572(42)$ \\
Facial palsy & + & $80 / 121(66)^{\mathrm{a}}$ & $15 / 519(3)$ \\
Motor delay & + & $147 / 149(99)^{\mathrm{a}}$ & $557 / 641(87)^{\mathrm{a}}$ \\
Tracheoesophageal anomaly & - & $42 / 146(29)$ & $3 / 370(1)^{\mathrm{a}}$ \\
\hline
\end{tabular}

Frequency is shown as the number of patients with a feature/number of patients investigated. Data are based on information provided by Corsten-Janssen et al. [6].

+, present; -, not present; -, undetermined.

${ }^{a}$ Frequencies showing higher agreement with the features of our patient. 
receive $B C G$ vaccination before 1 month of age, an unusual reaction to the $B C G$ vaccine should alert clinicians about the possibility of primary immunodeficiencies. The severity of immunodeficiencies in patients with CHARGE syndrome ranges from mild to severe T lymphocyte deficiency and isolated humoral immunodeficiency, which is similar to that in patients with 22q11.2 deletion syndrome. Although functional analysis of T lymphocytes was not performed in our patient, most patients with CHARGE syndrome have decreased number and poorly functional T lymphocytes [14]. Oligoclonal expansion of poorly functional T lymphocytes results in normal or mildly decreased number of T lymphocytes, which may impede the diagnosis of immunodeficiency [14]. Isolated humoral immunodeficiency is rare. Low lgG level in our patient may reflect impaired number and decreased function of CD4 cells. Although the immune system of our patient was not severely affected, IgG deficiency along with velopharyngeal incoordination contributed to the recurrent and uncontrolled airway infections and finally death.

This is the first case of mutation-confirmed CHARGE syndrome with immunodeficiency and hypocalcemia in Korea. Before the availability of $C H D 7$ mutation analysis, patients with overlapping features of CHARGE and 22q11.2 deletion syndromes and concomitant immunodeficiency and hypocalcemia were likely to be diagnosed as having DiGeorge syndrome. Patients with overlapping features of CHARGE and 22q11.2 deletion syndromes should be suspected of having CHARGE syndrome, especially in the absence of chromosome 22q11.2 deletion, because these patients almost always show some degree of hearing loss, which requires appropriate auditory evaluation for accurate management [15]. In addition, immunological evaluation of all patients with CHARGE syndrome should be performed to estimate their immune function and to provide proper management for infectious illnesses.

In conclusion, clinical diagnosis of CHARGE and 22q11.2 deletion syndromes is challenging. Our observations suggest that mutation analysis of CHD7 should be performed for patients who have phenotypes of 22q11.2 deletion syndrome but do not have the chromosomal microdeletion. In addition, we propose that all patients with CHARGE syndrome should be evaluated for immunodeficiency and hypocalcemia.

\section{References}

1. Verloes A. Updated diagnostic criteria for CHARGE syndrome: a proposal. Am J Med Genet A 2005;133A:306-8.

2. Issekutz KA, Graham JM Jr, Prasad C, Smith IM, Blake KD. An epidemiological analysis of CHARGE syndrome: preliminary results from a Canadian study. Am J Med Genet A 2005;133A:309-17.

3. Zentner GE, Layman WS, Martin DM, Scacheri PC. Molecular and phenotypic aspects of CHD7 mutation in CHARGE syndrome. Am J Med Genet A 2010;152A:674-86.

4. Jongmans $M C$, Admiraal RJ, van der Donk $K P$, Vissers $L E$, Baas $A F$, Kapusta L, et al. CHARGE syndrome: the phenotypic spectrum of mutations in the CHD7 gene. J Med Genet 2006;43:306-14.

5. Lalani $S R$, Safiullah $A M$, Fernbach $S D$, Harutyunyan $K G$, Thaller $C$, Peterson LE, et al. Spectrum of CHD7 mutations in 110 individuals with CHARGE syndrome and genotype-phenotype correlation. Am J Hum Genet 2006;78:303-14.

6. Corsten-Janssen N, Saitta SC, Hoefsloot LH, McDonald-McGinn DM, Driscoll DA, Derks $R$, et al. More clinical overlap between 22q11.2 deletion syndrome and CHARGE syndrome than often anticipated. Mol Syndromol 2013;4:235-45.

7. Jyonouchi S, McDonald-McGinn DM, Bale S, Zackai EH, Sullivan KE. CHARGE (coloboma, heart defect, atresia choanae, retarded growth and development, genital hypoplasia, ear anomalies/ deafness) syndrome and chromosome 22q11.2 deletion syndrome: a comparison of immunologic and nonimmunologic phenotypic features. Pediatrics 2009;123:e871-7.

8. Kobrynski し, Sullivan KE. Velocardiofacial syndrome, DiGeorge syndrome: the chromosome $22 q 11.2$ deletion syndromes. Lancet 2007;370:1443-52.

9. McDonald-McGinn DM, Sullivan KE. Chromosome 22q11.2 deletion syndrome (DiGeorge syndrome/velocardiofacial syndrome). Medicine (Baltimore) 2011;90:1-18.

10. Rhim JW, Kim KH, Kim DS, Kim BS, Kim JS, Kim CH, et al. Prevalence of primary immunodeficiency in Korea. J Korean Med Sci 2012;27:78893.

11. Inoue $H_{1}$ Takada $H_{1}$ Kusuda $T$, Goto $T, O$ Chiai M, Kinjo $T$, et al. Successful cord blood transplantation for a CHARGE syndrome with CHD7 mutation showing DiGeorge sequence including hypoparathyroidism. Eur J Pediatr 2010;169:839-44.

12. Gennery AR. Immunological aspects of $22 q 11.2$ deletion syndrome. Cell Mol Life Sci 2012;69:17-27.

13. Kaliakatsos M, Giannakopoulos $A$, Fryssira $H$, Kanariou M, Skiathitou AV, Siahanidou T, et al. Combined microdeletions and CHD7 mutation causing severe CHARGE/DiGeorge syndrome: clinical presentation and molecular investigation by array-CGH. J Hum Genet 2010;55:761-3.

14. Writzl K, Cale CM, Pierce CM, Wilson LC, Hennekam RC. Immunological abnormalities in CHARGE syndrome. Eur J Med Genet 2007;50:338-45

15. Song MH, Cho HJ, Lee HK, Kwon TJ, Lee WS, Oh S, et al. CHD7 mutational analysis and clinical considerations for auditory rehabilitation in deaf patients with CHARGE syndrome. PLoS One 2011;6:e24511. 